\title{
A Rare Cause of Acute Abdomen: Radiotherapy- induced Bladder Rupture
}

\author{
Hülya Yılmaz Başer', Aykut Başer², Ali Ersin Zümrütbaş², Bülent Erdur' \\ 'Department of Emergency Medicine, Pamukkale University School of Medicine, Denizli, Turkey \\ 2Department of Urology, Pamukkale University School of Medicine, Denizli, Turkey
}

\begin{abstract}
Introduction: Spontaneous intraperitoneal rupture of the bladder, which is rare, is a life-threatening condition. In emergency services, when patients present with lower quadrant abdominal pain and acute renal failure in the patient's laboratory findings consistent with curriculum vitae should be suspected if there is a history of radiotherapy. A cystography is the most accurate and precise method to make a diagnosis. The definitive solution is surgery, which depends on the condition of the patient.

Case Report: A 52-years-old female patient with diffuse abdominal pain applied to our emergency department with complaints of high fever and hematuria. The fact that operated from cervical cancer medical history and undergone radiotherapy was learned. A physical examination of the abdomen and midline defects detected a urine-like liquid coming from the defect. Cystography was also performed because of being macroscopic hematuria to patient, and bladder rupture was detected.

Conclusion: The aim of this phenomenon in our presentation. If patients who present to the emergency department with acute renal failure and peritonitis with a medical history of radiotherapy have a history of pelvic radiotherapy in the curriculum vitae with acute renal failure and peritonitis emergency signs, spontaneous rupture of the bladder is to emphasize that occur years after radiation therapy should be considered.
\end{abstract}

Keywords: Spontaneous bladder rupture, pelvic radiotherapy, acute abdomen, acute renal failure

Received: 09.05.2015 Accepted:22.07.2015 Available Online Date: 04.02.2016

\section{Introduction}

Spontaneous bladder rupture is an uncommon clinical situation. Generally blunt is seen as iatrogenic, and penetrating trauma. Diagnosis and treatment; in reviewing cases overlooked or skipped, with potentially dangerous, requires urgent treatment. Spontaneous bladder rupture, infravesical obstruction, bladder tuberculosis, long-term cystitis, bladder diverticulum, bladder cancer after radiotherapy, and pelvic region as a result of weakening of the bladder wall can be seen. In this case, a patient who presented with abdominal pain and hematuria symptoms, which emphasized the importance of the systemic examination and questioning resumes in the emergency department after radiotherapy in the late period is bladder rupture.

\section{Case Report}

A 52-year-old female patient with diffuse abdominal pain applied to our emergency department with complaints of high fever and hematuria. For 10 days, the lack of oral intake, a decrease in urine output for 3-4 days, for the last $24 \mathrm{~h}$ on the patient's blood in the urine, indicating that for the last 3-4 hours, was intermittent abdominal pain and fever. Background in questioning; in 1999, he underwent an operation because of cervical cancer after operation without knowing the number of fractions and total dose. In 2000, radiotherapy was performed in the lower abdominal region for the disease. Four years ago, a transobturator tape was applied with complaints of urinary incontinence surgery.

On physical examination, rebound and defense were suspected to be positive. We found an approximately $0.5-\mathrm{cm}$ defect in the suprapubic midline abdominal incision scar, and this scar was inflamed (Figure 1). The patient's vital signs were as follows: fever, $39.8^{\circ} \mathrm{C}$ pulse, 97; respiratory rate, 18/min. Laboratory tests revealed the following: TA, 100/60 mmHg; Kre, $3.81 \mathrm{mg} / \mathrm{dL}$; BUN, 41

\section{Address for Correspondence:}

Hülya Yılmaz Başer, Department of Emergency Medicine, Pamukkale University School of Medicine, Denizli, Turkey

E-mail:ylmz_hly_35@yahoo.com

oCopyright 2016 by Emergency Physicians Association of Turkey - Available online at www.jemcr.org 


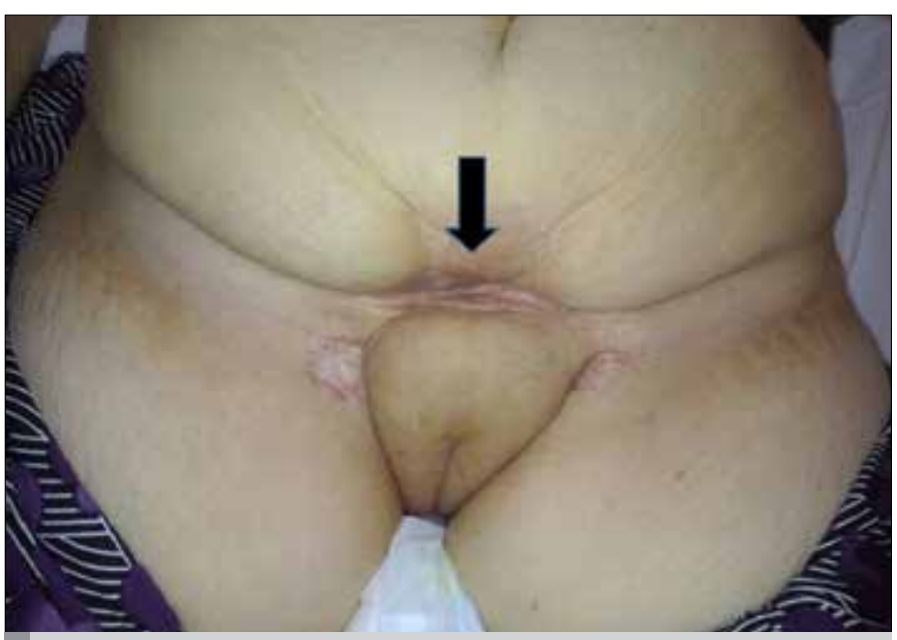

FIG. 1. Old incision scar in the suprapubic region (area indicated by the arrow)

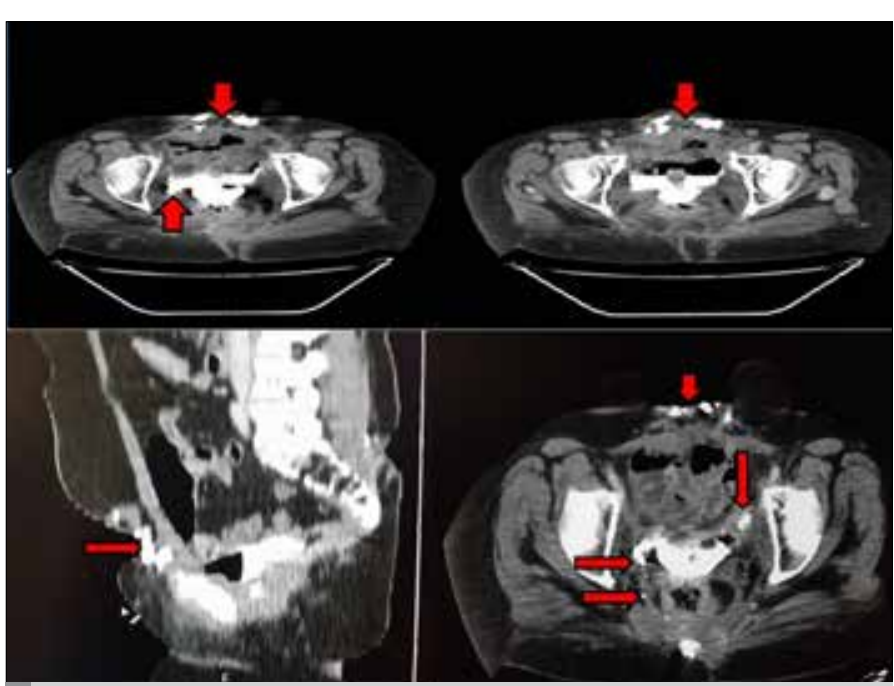

FIG. 2. Extralumination of contrast agent given in BT cystography, from the defect in the anterior bladder wall to the anterior abdominal wall and paravesical area (area indicated by the arrow)

mg/dL; Na: 133 mmol/L; K, 5.5 mmol/L; Cl, 97 mmol/L; INR, 1.55; Hb, $6.9 \mathrm{~g} / \mathrm{dL} ; \mathrm{Htc}, 22.7 \% ;$ MCV, 66.8\%, WBC, $17920 \mathrm{~K} / \mathrm{uL} ; \mathrm{PLT}, 377000 \mathrm{~K} / \mathrm{uL}$; TIT, 349 leukocytes and 257 erythrocytes; density, 1012; $\mathrm{pH}$ : :5.5; the blood gas; $\mathrm{pH}, 7.55 ; \mathrm{HCO}_{3}, 16.8 \mathrm{mmol} / \mathrm{L}$; and $\mathrm{SO}_{2}, 98.4 \%$. The patient underwent cold application and antipyretic treatment and was intubated because of macroscopic hematuria; a urology consultation was requested and a BT cystography was performed. The ekstraluminasyon of contrast agent given in BT cystography, which bladder defect in the abdominal wall toward the front wall and paravezikal field, was followed (Figure 2).

Ertapenem was initiated to patients with signs of peritonitis. Two units of packed red blood cells and fresh frozen plasma were given to the patient who had a history of using warfarin sodium. The patient were internalized by urology, the patient was followed for probe, and was informed about the follow-up and surgical options.
The patient who did not accept surgical treatment due to the declining number of complaints after the follow-up probe was discharged with the probe. The patient whose complaints repeated after 6 months, cure was achieved after surgery.

\section{Discussion}

Radiotherapy to the pelvic region can be applied to several types of cancer; however, adjacent organs such as the bladder, ureter, and rectum are exposed to the serious side effects of treatment. Following curative radiotherapy, the rate of serious side effects developing in primary cancer has been reported to be approximately $10 \%$ and for urological complication, it is approximately $3 \%$, and the rate depends on the dose of radiotherapy, fraction of the dose, and volume of irradiation (2). Pathological changes in the bladder of radiotherapy is a wide range. Abnormalities such as inflammatory infiltrates, fibrosis, cellular atypia, and necrosis, can cover the entire wall of the bladder and can also lead to vascular pathologies. These changes can lead to spontaneous perforation as a result of structural weaknesses in the bladder, and they are also considered to result in the formation of hematuria, fibrosis, cystitis, contractions, and fistula $(2,3)$.

In adults, following radiotherapy, the timing of these perforations was reported to be a period of approximately 15 years. Although repeat episodes are known, they should be treated as acute when detected (4). In our case, this time has been seen as about 13 years and it is similar to literature.

In English literature, the first case of spontaneous bladder rupture associated with pelvic radiation was reported by Altman and Horsburg in 1966 (5). The incidence of spontaneous bladder rupture is unclear (4). Fujikawa et al (1) reported the rate of spontaneous bladder rupture to be approximately $2 \%$ in 271 Japanese patients with radiotherapy complications.

The most common location for intraperitoneal perforation is the dome or the posterior bladder wall. This situation is explained by the fact that the posterior bladder wall or dome is the weakest point. In our case, it was detected in the perforation of the bladder anterior wall. The reason for this is probably trauma to the anterior bladder wall in the operation for cervical cancer or radiotherapy irritated after denser in this area.

Intraperitoneal rupture is more common than extraperitoneal rupture. The classic sign of intraperitoneal rupture is a sudden pain in the suprapubic region, making it difficult to urinate. Although initial findings are minimal in the beginning, they may reveal the signs of peritonitis on physical examination. We also detected the signs of peritonitis in our case. Cystography is the most accurate and precise method in the diagnosis of bladder rupture (6). BT and USG contrast are the other diagnostic methods, but they are not as sensitive as cystography.

Laboratory findings may show us spontaneous bladder rupture similar to radiological diagnostic methods. As a result of reabsorption through the peritoneum of urea and creatinine spreading from 
the bladder to peritoneum, blood, urea, and creatinine levels significantly rise. Due to the similarity between acute renal failure and many of these laboratory findings, intraperitoneal bladder rupture is difficult to identify (7). We identified similar laboratory findings in our case, and we thought acute abdomen and a preliminary diagnosis of $A B Y$ in the first place.

Although the mortality rate is as high as $25 \%$ in the diagnosis passing $24 \mathrm{~h}$, it can be minimized with proper diagnosis and immediate surgical correction (8). In our case, no surgical operation was performed because the patient refused surgical intervention and the number of complaints with follow-up with probe declined.

In conclusion, in a patient presenting with complaints of fever, abdominal pain, and hematuria to the emergency department in the presence of creatinine elevation and peritonitis symptoms, bladder rupture should be suspected if the patient has a pelvic radiotherapy history.

Informed Consent: Written informed consent was obtained from patient who participated in this study.

Peer-review: Externally peer-reviewed.

Author contributions: Concept - H.Y.B., A.B., A.E.Z.; Design - H.Y.B., A.B., B.E.; Supervision - H.Y.B., A.B., A.E.Z.; Resource - H.Y.B., A.B., B.E.; Materials - H.Y.B.,
A.B., A.E.Z.; Data Collection and/or Processing - H.Y.B., A.B., B.E.; Analysis and/ or Interpretation - H.Y.B., A.B., A.E.Z.; Literature Search - H.Y.B., A.B., B.E.; Writing - H.Y.B., A.B., B.E.; Critical Reviews - HY.B., A.B., A.E.Z.

Conflict of Interest: The authors declared no conflict of interest.

Financial Disclosure: The authors declare that they don't receive financial support for this work.

\section{References}

1. Fujikawa, K, Miyamoto T, Ihara Y, Matsui Y, Takeuchi H. High incidence of severe urologic complications following radiotherapy for cervical cancer in Japanese women. Gynecol Oncol 2001; 80: 21-3. [CrossRef]

2. Covens A, Thomas GM, Depetrillo AJ, Jamieson C, Myhr T. The prognostic importance of site and types of radiation induced bowel injury in patient requiring surgical management. Gynecol Oncol 1990; 43: 270-4. [CrossRef]

3. Baseman AG, Snodgrass WT. Repeat spontaneous bladder rupture following radiation therapy. J Urol 2003; 170: 2417. [CrossRef]

4. Suresh UR, Smith VJ, Lupton EW, Haboubi NY. Radiation disease of the urinary tract: histological features of 18 cases. J Clin Pathol 1993; 46: 228-31. [CrossRef]

5. Atman B, Horsburgh AG. Spontaneous rupture of the bladder. $\mathrm{Br} J$ Urol 1966; 38: 85-8. [CrossRef]

6. Carroll PR, McAninch JW. Major bladder trauma: the accuracy of cystography. J Urol 1983; 130: 887-8.

7. Vohra SB, Kapur S. Diagnosis of bladder rupture by arterial blood gas analysis. Anaesthesia 2007; 62: 534-5. [CrossRef]

8. Shaked A, Merrtyk S, Pode D, Caine M. Non traumatic spontaneous rupture of the urinary bladder. Can J Surg 1986; 29: 107-9. 